\title{
Thesen zur Ir/Rationalität des Geldes
}

Jochen Hörisch

Der folgende Text ist die Transkription eines frei gehaltenen Vortrags.

Vielen Dank für die Einladung, die hochgradig willkommene! Sehr geehrte Damen und Herren, man sagt es immer so konventionell - aber ich bin wirklich dankbar, weil es so selbstverständlich gar nicht ist, dass man einen Germanisten einlädt zu einer Tagung mit diesem Thema. Ich will Ihnen daher gleich zu Beginn die recht abstrakte Überlegung zumuten, warum man möglicherweise zu diesem Thema als Literaturwissenschaftler etwas zu sagen hat.

Eine anekdotische Antwort, oder mehr als eine anekdotische Antwort wäre: Ihr verehrter Vater, lieber Herr Binswanger, hat mit seiner Faust-Interpretation ${ }^{1}$ mein Fach beschämt - beschämt, weil keinem Germanisten die hohe Plausibilität der Interpretation von Hans Christoph Binswanger aufgefallen ist. Seine Anregung war ungeheuer: Wir haben bei den ganzen turns in den Geisteswissenschaften - wir sind ange->turnt< durch den colonial, gender, spatial oder iconic turn - den entscheidenden Dreh übersehen. Doch der economic turn ist nicht nur in der Germanistik, sondern in der Literaturwissenschaft überhaupt, der produktivste. Er war auch ein Schub, der uns genötigt hat, heilige Texte wie Goethes Faust anders zu lesen. Die große These von Hans Christoph Binswanger war ja, dass Goethe die großartige Vermutung formuliert, dass Geld der Sphäre der Alchemie sehr nahe ist und man schlecht beraten sei, wenn man versucht, ökonomische und vor allen Dingen finanzökonomische Prozesse mathematisch oder am Paradigma des Physikstudiums zu entwickeln, sondern dass wir beim Geld in einer Sphäre einer irrationalen Zauberei sind, die produktive Nebeneffekte freisetzen kann. Wir alle wissen, dass wir der Alchemie beispielsweise das Porzellan zu verdanken haben. Also: Es kann aus Falschheiten und Verrücktheiten etwas Produktives, Konstruktives, eine technische Innovation - Herr Deutschmann, um mit Ihnen zu sprechen ${ }^{2}$ - entstehen. Das wäre die anekdotische Geschichte, die uns am Beispiel der Binswanger- 
Interpretation gezeigt hat, dass wir Germanisten wirklich geldblind waren, obwohl es so überaus evident ist, dass der ganze Faust um Geldthemen kreist: "Nach Golde drängt/Am Golde hängt/Doch alles! Ach, wir Armen « ${ }^{3}$ - vor allen Dingen in Faust II, erster Aufzug. ${ }^{4}$

So viel zum Anekdotischen. Die zweite Überlegung ist weitaus abstrakter: der Untertitel unserer Tagung verweist auf die kollektive Wahrnehmung von Problemen, die mit der Geldsphäre verbunden sind. Und da ist natürlich das Eigentümliche, dass - wenn man Literatur als ein Medium des Wissens begreift - wir es hier mit einer hochgradig nicht-kollektiven, sondern vielmehr mit einer individualisierten, einer idiosynkratischen, ja, hochgradig unplausiblen Wahrnehmung zu tun haben. Literatur, die sschöne Literatur , ist ja nicht verpflichtet, die Wahrheit zu sagen - im Gegenteil: Literatur hat die Lizenz zur Lüge, Literatur hat die Lizenz zur Fantasie, zum abwegigen Gedanken, also genau zu dem, was der kollektiven Wahrnehmung und eingespielten Verständigungsfiguren widerspricht. Ich mute Ihnen ganz schlicht - aber Sie kennen das ja schon - die systemtheoretische Grundsatzüberlegung zu, dass wir in ausdifferenzierten, modernen Gesellschaften Teilsysteme haben, die jeweils auf ein Problem spezifisch reagieren, nämlich mit einem binären Code und autopoietisch. ${ }^{5}$ Die Alternative wäre, dass man ein hierarchisiertes, stratifiziertes System hat, dessen Spitze glaubt, für alle Probleme zuständig zu sein. Um das geschwind durchzudeklinieren: Es gibt Konflikte, die Leute streiten sich, ein Maschendrahtzaun wird zwischen Nachbarn gezogen. Weil es Konflikte gibt, brauchen wir ein Justizsystem, das mit der binären Codierung > Recht $<$ und >Unrecht $<$ arbeitet. Und wenn die Angeklagte, die den Liebhaber umgebracht hat, eine schöne Angeklagte ist, dann darf der Richter gerade eben nicht den ganzen Menschen im Blick haben und sagen: »Ich spreche sie frei, weil sie so unwiderstehlich schön ist «, sondern: »Sie hat ihren Lover umgebracht, das ist verboten, der ganze Mensch kann bei diesem Urteil keine Rolle spielen«. Oder: »Sie spielt so wunderbar die Mondscheinsonate, deshalb erlasse ich ihr fünf Jahre vom Strafrahmen«. Nein, so geht das nicht in modernen ausdifferenzierten Gesellschaften - und das ist auch gut so. >Autopoietischく heißt, es sprechen nur die Paragraphen der Justiz dafür, ob die Frau jetzt ins Gefängnis kommt oder nicht, ob sie schuldig oder nicht schuldig ist. Also: Ohne Probleme keine Systeme. Konflikte sind das Problem, die Justiz das es bearbeitende System. Knappheit ist ein

3 Goethe, Johann Wolfgang von: Faust. Der Tragödie Erster Teil, in: Ders., Faust. Texte (= Frankfurter Ausgabe, Band 7,1), herausgegeben von Albrecht Schöne, Frankfurt a.M.: Deutscher Klassiker Verlag 1994, S. 33-199, hier V. $654 \mathrm{ff}$.

4 Vgl. Goethe, Johann Wolfgang von: Faust. Der Tragödie Zweiter Teil, in: Ders., Faust. Texte (=Frankfurter Ausgabe, Band 7,1), herausgegeben von Albrecht Schöne, Frankfurt a.M.: Deutscher Klassiker Verlag 1994, S. 201-464.

5 Vgl. Luhmann, Niklas: Soziale Systeme. Grundriss einer allgemeinen Theorie, Frankfurt a.M.: Suhrkamp 1984. 
Problem, die Wirtschaft das sich an ihm abarbeitende System. Ihr binäres Schema ist »kaufen«/»nicht kaufen«; »zahlen«/»nicht zahlen«. Das Wirtschaftssystem spezialisiert sich auf Bewältigung von Knappheiten und versucht das spezifisch mit diesem Code »zahlen«/»nicht zahlen« zu machen. ${ }^{6}$

Das Religionssystem wiederum arbeitet sich ab am Problem der Kontingenz. Warum bin ich 1951 und nicht zur Goethezeit geboren? Warum als Mann und nicht als Frau? Es gibt zigtausend Antworten auf die Problemfrage: »Warum ist überhaupt Seiendes und nicht vielmehr Nichts?«. Lauter Fragen, die schwer zu beantworten sind, weil sie das Problem der kleinen oder der absoluten Kontingenz fragen. Darauf spezialisiert sich das Religionssystem, und das macht es, indem es mit der Unterscheidung arbeitet: »immanent «/»transzendent $«{ }^{7}$ Mir hat diese These Niklas Luhmanns immer sehr eingeleuchtet. Der Pfarrer darf eben in aufgeklärten Gesellschaften dem Wissenschaftler nicht reinreden und Vorschriften machen. Er kann zwar sagen: »Was Du als Wissenschaftler machst, hat enorme Folgen für die Glaubensbereitschaft der Leute.« Er darf aber nicht sagen: »Einstein, Du hast Unrecht, weil das alte theologische Weltbild kollabiert.« Und die große Rätselfrage ist: Wenn jetzt Kunst und Literatur auch ein System sind - man kann das ja infrage stellen, aber ich denke, sie sind auch ein System -, das systematische Leistungen erbringt, was ist dann das Problem, auf das Kunst, Literatur, und so weiter, Musik, Bildende Kunst reagieren? Es ist das Problem der Vieldeutigkeit. Man kann etwas so oder anders sehen. Das Glas ist halb voll oder halb leer. Das sind sachlich beides haltbare Aussagen, aber für die Lebenshermeneutik macht das eben einen Unterschied. Und die binäre Codierung, mit der das Kunstsystem auf solche Vieldeutigkeits-Probleme reagiert, ist die von »kohärent«/»inkohärent«, "gut gesagt«/»schlecht gesagt«, »stimmig«/»nicht stimmig«. »Ist das Metrum in Ordnung?«, »Ist das ein Reim, der uns überrascht?«.

»Die schönsten Gedichte der Menschen,/- nun finden Sie schon einen Reim!«, dichtet Peter Rühmkorf und umkreist damit das Problem, warum es in der deutschen Sprache auf >Mensch $<$ kein Reimwort gibt. Dann kommt Rühmkorf und hat diese geniale Idee zu sagen: »Die schönsten Gedichte der Menschen,/- nun finden Sie schon einen Reim! -/sind die Gottfried Bennschen. ${ }^{8}$ Und dann kommen Verse, die Schleim, sinkende Euphrat-Throne und Epigonen umkreisen. Ihm gehen die poetischen Pegasus-Pferde durch - und wir alle gehen auf die Knie und sagen: "geistreich, gut gesagt. "Ob das sachlich stimmt, steht auf einem ganz anderen und weniger wichtigen Blatt. Um die übliche Provokation zu verwenden:

6 Vgl. Luhmann, Niklas: Die Wirtschaft der Gesellschaft. Frankfurt a.M.: Suhrkamp 1988.

7 Vgl. Luhman, Niklas: Die Religion der Gesellschaft, herausgegeben von Andrè Kieserling, Frankfurt a.M.: Suhrkamp 2000.

8 Rühmkorf, Peter: Irdisches Vergnügen in g, Reinbek: Rowohlt 1959, S. 60. 
Wenn Gottfried Benn dichtet: »Der soziologische Nenner,/der hinter Jahrtausenden schlief,/heißt: ein paar große Männer/und die litten tief «, ${ }^{9}$ sag nicht nur ich allein: »das ist ja fürchterlich politisch inkorrekt, wissenschaftlich nicht haltbar, absoluter Blödsinn«, aber es gibt da eine Ecke in unserem Herzen, wo wir sagen: "Der Sound ist gut, bekämpft das in der Sache, aber bewahrt euch ein bisschen Respekt vor dem Einfall, vor der These, vor dem Sound, vor dem Gottfried-BennSound dieser Geschichte.«

Worauf will ich hinaus? Hochgradig idiosynkratische Wahrnehmungen, schwer haltbare Thesen, abweichende Wahrnehmungen, keine kollektiven, sondern gerade eben etwas, was der kollektiven, der eingefahrenen Wahrnehmung radikal widerspricht, macht Kunst so interessant und zu so einem spannenden Subsystem von Gesellschaften. Die Leistung, die Kunst im Allgemeinen erbringt, besteht darin um im Luhmann-Slang zu sprechen -, dass man alternative Realitätswahrnehmungen kopräsent hält zu den eingespielten. ${ }^{10}$ Auch eine extreme Unwahrscheinlichkeit, wie dass der soziologische Nenner ein paar große Männer sind, wird als Denkmodell, auch wenn es falsch ist, weiter kopräsent gehalten, weil es gut gesagt ist. Man kann sagen, der Preis ist zu groß, wenn man das durchhält, aber das will ich jetzt gar nicht vertiefen.

Abweichende Wahrnehmung: Das, was Goethe im Faust über Geld zu sagen hat, über seine Rationalität und Irrationalität, über die emotionalen, theologischen und sexuellen Dimensionen des Geldes, widerspricht all dem, was ein Betriebswirt, was ein Volkswirt, was ein Soziologe in aller Regel - es sei denn, er ist so originell wie Deutschmann ${ }^{11}$ - über Geld ausführen würde. Es entspricht nicht dem, was man im Einführungskurs Soziologie, BWL oder VWL hört. Das ist auch nicht der Sinn literarischer Äußerungen über Geld, sondern, ich wiederhole mich: dagegen zu halten und eine absolut unwahrscheinliche Wahrnehmung gegen eine Lehrmeinung $\mathrm{zu}$ halten (und hinterhältig darauf hinzuweisen, dass sich BWL und VWL sinnvollerweise nicht als Wissenschaften, sondern als Lehren bezeichnen). Ich will meine von Goethe und Binswanger inspirierte These zur Irrationalität des Geldes illustrieren. Wir haben es alle noch im kollektiven Gedächtnis: Daimler und Chrysler fusionieren, und der damalige Vorstandssprecher von Mercedes, Schrempp, gibt ein legendäres Interview, in dem er sagt: »Was wir mit der Fusion von Daimler und Chrysler machen, ist eine Hochzeit im Himmel «. ${ }^{12}$ Da klingen natürlich beim Philologen alle Glocken: Hochzeit ist ein erotischer, emotional besetzter Begriff,

9 Benn, Gottfried: Sämtliche Werke. Band I. Gedichte 1, herausgegeben von Gerhard Schuster, Stuttgart: Klett-Cotta 2007, S. 174.

10 Vgl. Luhmann, Niklas: Die Kunst der Gesellschaft, Frankfurt a.M.: Suhrkamp 1997.

11 Vgl. z.B. Deutschmann, Christoph: Die Verheißung des absoluten Reichtums. Zur religiösen Natur des Kapitalismus, 2., überarbeitete Auflage, Frankfurt a.M./New York: Campus 2001.

12 Vgl. z.B. Bertram, Brigitte/Jocham, Andrea: »Ehe auf Rädern«, in: Handelsblatt vom 07.05.2003, http://www.handelsblatt.com/archiv/fuenf-jahre-daimler-chrysler-ehe-auf- 
und Himmel ist ein theologischer Begriff. Da haben wir also einen Spitzenmanager, der extrem mit sexualisierten und religiös aufgeladenen Begriffen arbeitet. Meine Kollegen in Mannheim aus der BWL und aus der VWL sagen: "Also bitte, wenn du das so wahrnimmst, völliger Blödsinn. McKinsey und andere haben die beraten, es gibt Skaleneffekte, die sparen so und so viel ein, es ist eine absolut rationale Entscheidung«. Als Philologe würde ich umgekehrt sagen: »Man merkt schon an dieser Äußerung Schrempps: Da ist einer durchgeknallt, das ist ein hochgradig irrationales Investment.«

Leider hat der Philologe in dem Fall auch einmal Recht, war ja wohl keine so gute Idee, die Sache ist ziemlich dumm gelaufen. Aber wie gesagt, aus BWLSicht und auch aus volkswirtschaftlicher Sicht hieß es zumeist: ein joint venture, das sich lohnt, es rechnet sich, es ist darstellbar und dergleichen mehr. Dies nur, um deutlich zu machen, wie weit man möglicherweise auch diagnostisch und prognostisch kommen kann, wenn man abweichenden, aber literarisch alimentierten Wahrnehmungsmodellen verpflichtet ist. Und insofern freue ich mich, lieber Herr Deutschmann, natürlich auch sehr, dass Sie den Begriff der Fiktion mit verwendet haben. ${ }^{13}$ Dem Literaturwissenschaftler fällt wiederum auf, dass mehrere Begriffe aus der Finanz- und aus der Börsensphäre genuin poetische Begriffe sind. Ich denke, wenn ich zu einer Bank gehe und frage: »Könnt Ihr mir eine Hypothek geben?«, dann rechnen die das durch und wollen ein paar Unterlagen haben, und die Antwort ist dann: "Das ist darstellbar, oder nicht darstellbar«. Das ist doch offenbar ein Banker-Begriff, nicht? Die Darstellbarkeit der Kreditanfrage. Darstellbarkeit ist aber natürlich ein ästhetischer Begriff. Kann man es darstellen? Wie kann man es darstellen? Wenn Sie jung sind und wollen an die Börse gehen, dann brauchen Sie eine Story. Eine Story, mit der Sie beim Börsengang mögliche Kunden einigermaßen überzeugen können. Und heute ist das Wort `Narrativ « die Europäische Union braucht ein neues Narrativ, weil sie in der Krise ist und so weiter - in aller Munde. Es ist ein Modewort, aber ja ein außerordentlich erfolgreiches und verbreitetes Modewort.

Die hohe Frequenz ästhetischer Begriffe im Finanzsektor ist schon auffallend. Sie gibt dem Vertreter eines Faches, das aus gutem Grund legitimieren muss, warum es von Steuerzahlern alimentiert wird, Selbstvertrauen. Zum Teufel, wozu brauchen wir Fächer wie Literatur- und Kulturwissenschaft? Brauchen wir die wirklich? Und natürlich wäre meine Antwort: »Ja. «Wenn denn die Literaturwissenschaft so gut beraten ist, etwa Binswanger zu lesen und das Potential an Wissen, das in Kunst angelegt ist, auch wirklich abzurufen, thesenförmig zu machen, kompatibel zu machen mit konkurrierenden, wissenschaftlichen Einwänden. So

raedern/2244874.html?ticket=ST-2757397-thoo6FuY9bn5SSQkDSDN-ap3 (letzter Abruf 20. 06.2020).

13 Vgl. den Beitrag von Christoph Deutschmann in diesem Band. 
viel als abstrakte Vorbemerkung methodischer Art. Ich hoffe, ich werde nicht gleich unterbrochen und rausgeworfen aus der Tagung, weil ich bewusst nicht auf kollektive Wahrnehmung, sondern auf kritische, literarische, ästhetische, abweichende Wahrnehmungen setze. Das gilt auch für den Theorieteil, den ich jetzt einschalten möchte.

Die beiden Mega-Diagnosen dessen, was in der, ich sage mal getrost: bürgerlichen Moderne geschieht, stammen von Max Weber und Karl Marx. Da wäre die berühmte Max Weber-Diagnose, dass wir in der Moderne in einem sstählernen Gehäuse leben. ${ }^{14}$ Stählernes Gehäuse - das heißt: funktionierende Verwaltung, rechenhaftes Wirtschaften, Walten von Sachzwängen aller Art. Das alles ist kalkuliert und kalt, es bleibt kein Platz für die großen Gefühle und den Heroismus. Das alles ist gut so, aber es ist zugleich eine große Zumutung. So lautet bis hin zu dem Kultbuch von Helmut Lethen Verhaltenslehre der Kälte ${ }^{15}$ die verbreitete Diagnose über das, was uns in der Neuzeit und Moderne zugestoßen ist. Wir überleben im Zeitalter der Verdinglichung, der Entfremdung, der Rechenhaftigkeit, der Kalkulierbarkeit, des stählernen Gehäuses. Die Gegendiagnose, das macht man sich eigentlich selten klar, ist schon ein paar Jahrzehnte vor Max Weber ausgesprochen worden, sie stammt von keinem anderen als von Karl Marx, dessen zweihundertsten Geburtstag wir ja im letzten Jahr gefeiert haben. Marx hatte ein sehr feines Sensorium dafür, dass der Kapitalismus gerade keine Rationalitätsveranstaltung ist, sondern die inkarnierte Irrationalität darstellt, die verkehrte Welt, die man vom Kopf auf die Füße stellen muss. Wie in einer Camera Obscura ist im Kapitalismus alles verdreht und verrückt, nicht an seinem Platz - so lautet Marxens Gegendiagnose. Die Moderne ist das Zeitalter - ich zitiere aus dem Kapital - »der theologischen Spitzfindigkeiten und der metaphysischen Mucken ${ }^{16}{ }^{16}$ die in der Warenund in der Geldform stecken. Die Moderne ist verzaubert, sie ist verrückt, sie ist magisch, sie ist irrational, die Vormoderne war mit ihrem Ordo-Denken hingegen rational gegliedert und verlässlich. Mir hat das immer eingeleuchtet. Denken Sie einfach daran, wie ökonomisch geregelt und durchkalkuliert vormoderne Hochzeiten waren. In Gesellschaften, die wir als vormodern oder nicht richtig in der Moderne angekommen wahrnehmen, gilt das ja immer noch. Sie gucken sich vormoderne, traditionelle Bräuche an - ich hoffe, dass ich nicht nur Klischees bediene, oder ich hoffe, dass ich nur Klischees bediene und dass alles faktisch ganz anders ist - und stellen fest, da spielen der Brautpreis und die rationalen Aushandlungen

Vgl. z.B. Weber, Max: »Die protestantische Ethik und der Geist des Kapitalismus«, in: Ders., Gesammelte Aufsätze zur Religionssoziologie I, Tübingen: Mohr Siebeck 1988, S. 17-206, hier S. 204.

Vgl. Lethen, Helmut: Verhaltenslehren der Kälte. Lebensversuche zwischen den Kriegen, Frankfurt a.M.: Suhrkamp 1994. 
zwischen Familien, die die Hochzeit arrangiert haben, also rationale Erwartungen und Einstellungen eine extrem große Rolle.

Liebesheiraten sind ein Produkt der Moderne. Dass man sich die Verrücktheit einer Liebesheirat leisten kann, auch gegen den Widerstand des Herkommens, auch gegen ökonomische Überlegungen beider Familien, aus denen die Liebenden kommen, das ist ein Produkt der Moderne. Die Moderne kann sich Romantik leisten, weil sie so sehr auf Verrücktheit setzt und weil sie mit dem Setzen auf Verrücktheit, auf Irrationalität eine ungeheure Produktivität freisetzt. Ein anderes Beispiel: Gucken Sie sich den Umgang mit Tieren im Mittelalter und in vormodernen Gesellschaften an - war der liebevoller als er unter Tierschutzbedingungen heute ist? Sie gucken sich die Frauenemanzipation an, den Umgang mit Kindern, wer nimmt wen ernst? Ist ein empathischer Umgang miteinander im Spätmittelalter oder im frühen Mittelalter oder in der Antike häufiger oder ist das stärker in modernen Gesellschaften vertreten? Und wir merken, dass Sensibilität, Romantik, Gefühlswerte, Umweltbewusstsein und dergleichen mehr eben ein Produkt der Moderne sind. Die Moderne ist romantisch, sie ist sensibel in ihren besten Dimensionen. Dass es Gegentendenzen gibt, Faschismus, Krieg, und so weiter, brauchen wir hier nicht zu erörtern, aber dass romantische Ideen als regulative Ideen in die Welt kommen, denen sich so gut wie alle mehr oder weniger verpflichtet fühlen, das ist eben das große Novum der Moderne.

Nun war Marx, wie Sie alle wissen, ein Hegelianer, der in seiner Geld- und Warenanalyse ja eine sehr, sehr weitreichende These entwickelt, nämlich die, dass im Geldverkehr selbst eine Irrationalität steckt. Warum? Weil das Geld interessanterweise ja gerade eben nicht rational in dem Sinne ist, dass es evidente Identitätsbeziehungen herstellt. 20 Ellen Leinwand $=1$ Rock, beide sind äquivalent, kosten dieselbe Geldsumme. ${ }^{17}$ Eine solche Gleichung bedeutet ja, dass man Dinge, die nicht identisch sind, gleichsetzt. Tautologien sind langweilig. Dieser Stein ist dieser Stein, dieser Pullover ist dieser Pullover, diese Brille ist diese Brille - das wirft analytisch wenig ab. Spannend ist vielmehr festzustellen und festzusetzen: »Diese Lesebrille kostet 10 Euro und dieser Besuch im Café mit der Liebsten kostet ebenfalls 10 Euro - beide Werte sind äquivalent.«Was hat die Brille mit dem Cafébesuch zu tun? Gar nichts. Beides wird aber über Geld äquivalent gesetzt. Wir können das Wort ‘äquivalent - ich bin wieder auf der Philologenseite - ins Deutsche übersetzen und sagen: »Es ist gleich-gültig." Es ist in der Tat eine coole Operation, die aber als coole Operation einen sehr heißen Kern hat, nämlich die Identifikation des nicht Identischen. Im Hegel-Slang gesprochen: »Es ist eine Identität von Identität und Differenz. ${ }^{18}$

17 Vgl. ebd., S. 63.

18 Abgewandeltes Zitat; für die entsprechende Textpassage vgl. Hegel, Georg Wilhelm Friedrich: »Differenz des Fichte'schen und Schelling'schen Systems der Philosophie«, in: Ders., Ce- 
Wir setzen im Geldverkehr gleich, was nicht gleich ist. Und ich denke - aber das wäre Nebenthema -, dass Leute wie Adorno und Sohn-Rethel, in gewisser Weise auch Simmel mit seiner Philosophie des Geldes ${ }^{19}$ einfach Recht haben, wenn sie darauf aufmerksam machen, dass wir im heißen Zentrum eines vermeintlich rationalen Geldverkehrs die Irrationalität einer systematischen Identifikation des definitiv Nichtidentischen haben - Identifikation natürlich im Sinne von Äquivalenz. Wenn man dann noch einen Schritt weiter geht und die Marx'sche Semantik ernst nimmt und das weiter spinnt, zum Beispiel mit einem bedeutenden Buch von Herrn Deutschmann, ${ }^{20}$ merkt man sehr schnell, wie viele theologische Momente in der Finanzsphäre stecken. Ich schlüpfe wieder in das Gewand des Philologen und mache ganz geschwind eine Werbeblock Einblendung und verweise auf meinen kleinen Essay Die Theologie der Märkte - Man muss dran glauben. ${ }^{21}$ Es fällt auf, wie viele theologische Begriffe die Geldsphäre grundieren und mit Konnotationen und vibrations versehen. Sie nehmen bei der Volks- und Raiffeisenbank einen Kredit auf, das kommt von credo, dann sind Sie Schuldner und haben in der Volksund Raiffeisenbank einen Gläubiger. Wenn Sie den Kredit deutlich und gut umsetzen, können Sie damit ein Produkt platzieren, auf einer Messe. Dann meinen Sie aber nicht mehr das katholische Hochamt, sondern eben die Industriemesse, wo Sie Ihre Innovation ausstellen und die ist dann erfolgreich. Wenn sie erfolgreich ist, dann gibt es eine Wertschöpfungskette, da ist der Schöpfungsbegriff mit drin. Wenn Sie nicht erfolgreich sind und pleite gehen, müssen Sie einen Offenbarungseid machen. Auch Banken müssen $\mathrm{ab}$ und an einen Offenbarungseid leisten - bei den seriösen Volks- und Raiffeisenbanken war das noch nie der Fall; es gibt aber das hartnäckige Gerücht, dass bei der Lehmann Brothers-Pleite ein Vorstandsmitglied der Deutschen Bank mit seinem Depot von der Deutschen Bank zur Volksund Raiffeisenbank gegangen ist. Ich habe das nicht verifizieren können, ich halte es aber für eine absolut plausible Geschichte. Klammer zu. Um unserem Gastgeber mit Überzeugung zu huldigen: Ich bin originellerweise als badischer Beamter bei der Badischen Beamtenbank, obwohl die ja glaube ich auch zum Verbund der Volks- und Raiffeisenbanken gehört. Ich kriege keine Provision für das, was ich gerade gesagt habe gerade, leider. Also, wir merken: Theologische Begrifflichkeiten finden sich in der Finanzsphäre zuhauf. Je höherstufig wir sind bei den Problemen, die die Geldsphäre umgeben, desto stärker ist die theologische Aufladung dieser Begriffe. Denken Sie etwa an den technischen Begriff des fiat money, das die EZB

sammelte Werke. Band 4 (= Jenaer Kritische Schriften), herausgegeben von Hartmut Buchner/Otto Pöggeler, Hamburg: Meiner 1968, S. 1-92, hier S. 64.

Vgl. Simmel, Georg: Phiolosophie des Celdes (= Gesamtausgabe Band 6), Frankfurt a.M.: Suhrkamp 2011.

20 Vgl. C. Deutschmann: Verheißung.

21 Vgl. Hörisch, Jochen: Man muss dran glauben. Die Theologie der Märkte, München: Fink 2013. 
schöpft. Sie schöpft Geld, ein Schöpfungsakt. Fiat lux, es werde Licht, sagt der Gott der Genesis. »Es werde Licht. Und es ward Licht«. »Es werde Geld. Fiat money«, sagt die EZB, und es wird Geld. Übrigens sind die Umstände dieser Geldschöpfung so konklavenhaft unöffentlich wie nur irgendetwas, keine Pressekonferenz, keine Beobachtung durch die Öffentlichkeit. Ich glaube, noch nicht einmal ein Protokoll, in dem mehr drin steht als »der Zinssatz wurde erhöht oder gesenkt « oder dergleichen mehr. Ein Satz, Schluss, aus, alle. Anders als in weiten Sphären demokratischer Gesellschaften ist die EZB-Sphäre eine Sphäre, die radikal der Beobachtung entzogen wird. Was da geschieht, darf keiner verraten, so wenig, wie man beim Konklave verraten darf, wem man seine Stimme als zukünftigem Papst gegeben hat.

Nun könnten Sie sagen: Aber hinter den theologischen Grundbegrifflichkeiten steckt ein sachlich-funktionales Konzept, das ersichtlich an der Religion orientiert ist. Wir alle wissen, dass wir die Geldillusion brauchen. Wenn Religion funktionieren soll, muss Gott beglaubigt werden; wenn das kollektiv nicht der Fall ist, klappen Religionen nicht. Und wir alle wissen, dass, wenn wir Geld nicht beglaubigen, die Geldillusion nicht beglaubigen, Banken kollabieren würden. Ich habe übrigens großen Respekt vor der politischen Sphäre, viele von den ein wenig Älteren werden auch das noch vor Augen haben, wie bei der Lehmann Brothers-Pleite damals noch Steinbrück als Finanzminister und Merkel als Kanzlerin aufgetreten sind und gesagt haben: „Wir beide garantieren Euch: Eure Einlagen sind sicher.« Für diesen Sprechakt gab es keine institutionelle Legitimation. Zwei individuelle Personen in hohen Ämtern können das nicht garantieren. Sie haben nicht das Instrumentarium, und, und, und. Es hat komischerweise geklappt, es waren Worte, die beglaubigt wurden und die wahrscheinlich eine mittlere Katastrophe, wenn nicht eine große Katastrophe verhindert haben. Es waren Zauberworte. Man muss daran glauben, und wenn man nicht daran glaubt, dann hätte man eben eine Finanzund Wirtschaftskrise vom Allerfeinsten gehabt. So hatten wir nur eine sehr ordentliche, aber unter uns gesagt, für Leute, die gut situiert sind, für unsereins also, handelte es sich um eine erstaunlich gut gelaufene Krise - gerade auch im Vergleich zu 1929. Auch wenn Sie fragen: »Wie ist Geld gedeckt? Wie ist das, was der Pfarrer in der Kirche erzählt, gedeckt? « Durch eine heilige Schrift und so weiter. Wir alle wissen, dass das nicht sehr seriöse wissenschaftliche Begründungsund Falsifikations- oder Verifikationsprogramme sind. Auch da haben wir also eine ganz, ganz starke Parallele zwischen Ökonomie und Religion. Die stärkste Parallele ist wohl die Hand-Metaphorik. Wir sind, wenn wir gläubig sind, alle in Gottes Hand. Gott ist allmächtig, wir durchschauen seine höhere Intelligenz nicht, wir sind nicht auf seinem Niveau, aber wir haben eben >Gottvertrauen<. Wir können Bonhoeffers Wort - »nicht tiefer fallen, als in Gottes Hand«. Die Gotteshand hält alles zusammen und die faustische Frage, was die Welt im Innersten zusammenhält, kann man dann eben sehr eindeutig beantworten, eben mit Gott. Die Neuzeit 
stellt von der Hand Gottes auf die invisible hand des Marktes um, der eine Superintelligenz ist, der wir kleinen Konsum- und Produktionssubjekte nicht das Wasser reichen können. Der Markt weiß alles, alles ist schon eingepreist, das ist eine Superintelligenz, auf deren Höhe sind wir nicht. Joseph Vogl spricht sehr schön von der Parallele zwischen der Theodizee-Frage: ${ }^{22}$ Wenn der liebe Gott allmächtig und ein guter Gott ist, wie kann dann das Erdbeben von Lissabon stattfinden? Wenn der Markt alles weiß, wie kann dann die Oikodizee-Frage beantwortet werden? Also parallel zur Theodizee-Frage. Es gibt Krisen, ja, was sich der liebe Gott, was sich der Markt dabei denkt, wir wissen es nicht, aber in the long run wird es schon wieder zu stabilisierten Gleichgewichtszuständen kommen, so die religiöse und so die ökonomische Antwort.

Ein weiterer Punkt, den ich für den allerheißesten halte: Wenn man Kapitalismus und Geldwirtschaft als irrational religiöse Veranstaltung begreift, so kreist diese Veranstaltung eindeutig um den Prozess der Wandlung. Und ich verwende ganz bewusst dieses Wort aus der Theologie im Lateinischen: Transsubstantiation. Wenn Sie gut katholisch sozialisiert worden sind, ist das Wort Ihnen vertrauter als Protestanten: Klingelingelingeling, und aus dem Esspapier ist dann der Leib Christi geworden und aus dem Wein eben das Blut Christi. Das ist ein Transsubstantiations-, ein Wandlungsakt, den viele so recht nicht mehr beglaubigen wollen. Nicht zuletzt an der Frage nach dem Abendmahl und wie es beglaubigt werden soll, haben sich Protestantismus und Katholizismus dann entscheidend unterschieden. Aber, wie auch immer, gerade wenn diese Diskussion stattfindet, setzt sich ein neues Medium, das beglaubigt wird, universal durch als ein Tausch und als ein ökonomische Prozesse zusammenhaltendes Medium, das Geldmedium. ${ }^{23}$ Und das große Versprechen des Geldmediums ist: "Die Wandlung klappt.« Das ist uns so selbstverständlich geworden, dass wir den großen Zauber, den großen magischen Zauber, der vom Geld als Wandlungsmedium ausgeht, gar nicht mehr wahrnehmen. Sie alle haben heute schon Wandlungen vollzogen, weil Sie eben im Hotel eingecheckt haben oder einkaufen gewesen sind oder bei der Fahrt mit dem Bus hierher bezahlt haben und dergleichen mehr. Und auf einmal kriegen Sie ein Hotelzimmer und Sie kriegen eine Busfahrt und Sie kriegen ein Essen und so weiter. Das heißt, Sie schieben etwas Symbolisches rüber, keine Hostie, sondern ein Stück Papier, das bedruckt ist oder Sie zahlen mit Ihrer Unterschrift, oder, oder, oder und es kommt ein wertvolles Gut, eine wertvolle Dienstleistung dabei heraus: eine Form der Wandlung, die buchstäblich funktioniert. Das scheint mir der eigentliche Zauber des Kapitalismus zu sein.

Dass der kapitalistische Wandlungszauber nicht selbstverständlich ist, sieht man ja immer wieder. Blicken wir nach Venezuela, dann weiß man, was es heißt,

22 Vgl. Vogl, Joseph: Das Gespenst des Kapitals, Zürich: Diaphanes 2010.

23 Vgl. Hörisch, Jochen: Kopf oder Zahl. Die Poesie des Celdes, Frankfurt a.M.: Suhrkamp 1996. 
wenn dieses Wandlungsversprechen nicht mehr funktional ist. Dann gibt es Probleme, wie man sie bei Hyperinflationen hat. Vertrauen in die Wandlungskraft des Geldes ist eben nicht irgendeine Frage, an der das Funktionieren von Gesellschaften hängt, sondern die absolut entscheidende Frage. Also, invisible hand, Hand Gottes, Transsubstantiation, die wandelnde Kraft des Geldes, Theodizee, Oikodizee und die ganzen anderen Begrifflichkeiten, die ich genannt habe, sprechen kräftig mit, wenn man sagt: Es ist kein Wortgeklingel, es ist eine sachlich belastbare Strukturäquivalenz zwischen der Religionssphäre und der finanzökonomischen Sphäre vorhanden.

Ein weiterer Aspekt ist nicht minder emotional besetzt als glaubensintensive religiöse Fragen: Es ist die erotisch-sexuelle Dimension des Geldes. Man hat eine `Stange Geld , man hat ein erfolgreiches Unternehmen gegründet, aber die Bilanz knickt ein und dann muss man sie wieder aufrichten. Und wenn es gelingt, sie wieder aufzurichten, dann ist das Unternehmen potent. Eine völlig verbreitete Sprechweise, ein potentes Unternehmen. Woran merkt man, dass ein potentes Unternehmen da ist und nicht ein in Insolvenzgefahr geratenes Unternehmen? Weil es in der Lage ist, die Gläubiger zu `befriedigen . Der potente Unternehmer befriedigt den Gläubiger. Auch die große Vorstellung, dass Geld sich vermehren kann, ist sexy. Im Altgriechischen ist ja der Begriff für Geld unter anderem Token, was zugleich auch »Kinder« heißt. Es gibt Kinder und Kindeskinder, die hat man gemacht, man vermehrt sich, Geld vermehrt sich. So wie es Kinder und Kindeskinder gibt, gibt es Zinsen und Zinseszinsen. »Geld heckendes Geld «, ${ }^{24}$ würde Marx sagen. Es pflanzt sich fort, es vermehrt sich, es ist sexuell produktiv, es wird mehr. Also auch da merken wir, dass wir es sehr deutlich mit sexuellen vibrations in der Geldsphäre zu tun haben. Das gilt natürlich gerade in der Sphäre, die man in alter, linker Tradition das »ganz große Geld« nennt. Natürlich wissen wir alle, dass, wenn Sie nicht ordentlich alimentierter Beamter wie unsereins sind, in der erhabenen Geldsphäre andere Regeln gelten. Ich will übrigens der Letzte sein, der über höhere Beamtenbesoldung klagt, ich bin vielmehr sehr dankbar dafür, dass ich mein Berufsleben mit Ihren Steuerzahlerkosten verbringen konnte, auf eine Art und Weise, in der ich Leidenschaft und Hobby auf der einen Seite und Beruf auf der anderen Seite zusammenkriegen konnte - aber es gibt noch andere, wesentlich höhere Verdienstsphären als die eines deutschen Professors. Das schöne bzw. abgründige Problem beim großen Geld ist schnell benannt man merkt sehr schnell, dass das keine Entsprechung mehr haben kann auf der Realienseite, dass Sie nicht in bessere Restaurants oder Hotels gehen, Sie auch nicht mehr bessere Autos kaufen können oder auch die Möbel nicht mehr wertvoller sind, wenn Sie

24 Marx, Karl: Das Kapital. Dritter Band (= Marx Engels Werke, Band 25), Berlin: Dietz 1983, S. 405 . 
in der Sphäre sind, ob Sie, ich sage mal, 500.000 im Jahr machen oder eine Million. Und ob Sie eine Million machen oder fünf oder zehn Millionen im Jahr, wirkt sich ja auf den alltäglichen Lebensstandard nicht ansatzweise aus. Es ist dann entschuldigen Sie, wenn ich so männlich sexistisch spreche - wie bei Yachten. Ich habe nicht den Längeren, sondern die Längere.

Ich will jetzt nicht saltlinks auf Superreiche einschlagen, ich komme aus der Kurpfalz, wo wir sehr viele sehr beachtliche Mäzene haben, etwa aus dem Umkreis der SAP-Gründer, großartig, wie die mäzenatisch damit umgehen und etwa die neue Mannheimer Kunsthalle mal geschwind privat finanzieren und dergleichen mehr segensreiche Tätigkeiten ausgeübt haben. In dieser Sphäre spielt das symbolische Kapital eine entscheidende Rolle; es ist die pseudorationale Variante der Irrationalität des Geldes. Wer hat das teurere Bild zuhause hängen? Wer hat sich das kaufen können? Und natürlich weiß man, dass es nicht rational ist, wenn man für ein Bild, das Leonardo Da Vinci zweifelhafter Weise zugesprochen wird 450 Millionen zahlt, wie für »Salvator Mundi ${ }^{25}$ Das »Salvator Mundi«-Bild, Da Vinci zugesprochen, wurde in der Sektion für Gegenwartskunst versteigert. Warum? Weil kein einziges Farbsegment mehr aus der Zeit von Leonardo stammt, das ist $\mathrm{zu}$ Tode restauriert. Es ist auf Holz gemalt; das Holz ist authentisch aus der Leonardo-Zeit, das ist leicht heute rauszukriegen. Was macht den Wert von 450 Millionen aus? Eine Äquivalenz für eine erhabene Geldsumme finden! Es muss auch für Supervermögen im Bereich der wertvollen Dinge noch Äquivalente geben. Das scheint mir die wichtige Funktion, die magische, die verrückte Funktion des Kunstmarkts zu sein. Er erhält die Vision aufrecht, dass es Äquivalente zu Geld geben muss. Die gibt es aber sonst - ich wiederhole mich - im privaten Bereich nicht, es gibt nicht etwas, was man im Wert von einer Milliarde konsumieren kann. Aber da ist dann eben der hoch aufgeheizte, irrationale, magische, fetischistisch besetzte Kunstmarkt. Kein Wunder, dass Marx den Fetischbegriff, der ja sowohl ein religiöser als auch ein sexueller Begriff ist, rein kopiert hat in die Analyse von Finanzkapitalismus.

Ganz kurz nur als Hinweise für eine mögliche Dimension: Marx sagt: Der Kapitalismus ist verrückt. Er ist magisch. Er ist fetischistisch. Er zaubert. Er ist alchemistisch, mit Binswanger zu sprechen. Er ist nicht rational kalkulatorisch, sondern in seiner Basis wirklich von allerstärksten religiösen und sexuellen Impulsen her aufgeladen. Das hat enorme praktische Konsequenzen und Effekte. In der

25 Vgl. z.B. Zöllner, Frank: »Der teuerste Flop der Welt?«, in: Die Zeit vom 03.01.2019, htt ps://www.zeit.de/2019/02/salvator-mundi-leonardo-da-vinci-gemaelde-verkauf (letzter Abruf 20.06.2020). 
Polanyi-Perspektive ${ }^{26}$ will ich das andeuten, nicht ausführen. Es gibt drei Größen, die eigentlich nicht waren- und geldtauglich sind. Land wäre das erste Paradigma, das klassische Beispiel. Land ist in dem Sinne keine klassische Ware, dass man es nicht produzieren kann. Wenn wir großes Bedürfnis haben, nach Kühlschränken oder Autos oder Computern oder Smartphones, dann produzieren wir eben mehr Smartphones. Mehr Land können Sie (von kleinen, sophistischen Ausgestaltungen wie der am Ende von Faust II, 5. Akt einmal abgesehen) nicht so produzieren, wie man Smartphones produziert. Land wird aber wie eine Ware behandelt, die man für Geld bekommen kann. Bis heute ein aktuelles Problem. Wir schauen uns den Wohnungsmarkt und Betongeld an, das ist keine Kleinigkeit, was wir da als Problem haben. Ist Land eine Ware wie andere? Definitiv nicht. Sollen wir Land trotzdem so behandeln, als sei es eine Ware wie andere? Lange Diskussion, ich will sie nicht entscheiden. Zweitens das klassische Marx-Argument: Ist Arbeit eine Ware wie andere? Also kann man sagen, Mindestlohn, aha, Erhöhung von 8,40 auf 10,50. Eine Stunde Arbeitskraft ist dann soviel wert wie eine Kinokarte - wiederum die Äquivalenzsuggestion. Ist das, was Waren schafft, selbst als Ware behandelbar? Die Antwort ist: Ja, der Kapitalismus macht das. Aber ist das gewissermaßen koscher? Ist das eine Operation, die man analytisch, intellektuell, ethisch und so weiter rechtfertigen kann? Oder ist das eine Äquivalenzsetzung, die abgründige Dimensionen hat? Auch da will ich einfach nur das Problem in aller Knappheit exponieren. Der dritte Punkt ist eben das Geld selbst. Ist das Medium, das Tauschprozesse ermöglicht, erleichtert, Wandlungsprozesse ermöglicht, selbst wie eine Ware behandelbar? Kann man so tun, als sei Geld eine Ware wie andere Waren auch? Also: Land, Arbeit und Geld zu »commodifizieren«, um mit Polanyi zu sprechen, ist eine magische Grundoperation des Kapitalismus. Ich erwähne das nur, um deutlich zu machen, dass ich hoffentlich nicht bei philologischen Spintisierereien bin, sondern bei tiefen, strukturalen, sachlich relevanten Problemen, wenn ich den Kapitalismus mit Marx als verrückt erkläre.

Und jetzt kommt die Volte, die Sie überraschen mag, sie ist auch nicht ironisch gemeint, sondern mit Marx, zur Hälfte von mir aus auch von Hörisch, authentisch gemeint. Marx fängt ja das Kapital an mit einer großen Kapitalismushuldigung: "Der Kapitalismus ist eine ungeheure Warenansammlung ${ }^{27}$. Auch im Kommunistischen Manifest fällt er ja erstmal auf die Knie und betet die unglaubliche Produktivität des Kapitalismus im Vergleich zu anderen Wirtschaftsformen wie Merkantilis-

Vgl. Polanyi, Karl: The Great Transformation. Politische und ökonomische Ursprünge von Gesellschaften und Wirtschaftssystemen, übersetzt von Heinrich Jelinek, Frankfurt a.M.: Suhrkamp 1973. 
mus, Feudalismus usw. an. ${ }^{28}$ Und nicht ernsthaft bestreiten kann man ja bis heute, dass die Produktivität dieses magischen, irrationalen, finanzgesteuerten Kapitalismus ungleich größer ist als die etwa von zentralen Kommandowirtschaften, wie wir sie im Staatssozialismus haben. Es lässt sich dies nicht ernsthaft bestreiten. Und also würde ich Marx noch ein bisschen radikalisieren - und mich zugleich mit bestimmten Spätmarxisten anlegen wie mit meinen Kollegen in Mannheim aus der neoorthodoxen BWL- und VWL-Richtung - und sagen: Der Kapitalismus ist so erfolgreich, weil er mit der irrationalen, emotional unglaublich aufgeladenen Form des Geldes arbeitet und also bewusst auf Irrationalismus setzt. Der Kapitalismus ist produktiv aufgrund der Zulassung von sexuell und religiös aufgeladenem Irrationalismus - und weil er unkritisch ist. Er ist unkritisch gegenüber alten Tugendformen. Mit Marx zu sprechen - Lieblingszitat Ihres Vaters, lieber Mathias Binswanger: Faust taucht auf, Faust will wissen, wer Mephisto ist, und dann sagt Mephisto: »[Ich bin] [e]in Teil...« - Sie kennen es alle, bürgerliches Prunkzitat »...von jener Kraft,/Die stets das Böse will und stets das Gute schafft «. ${ }^{29}$ Das ist eine glatte Adam Smith-Paraphrase, private vices, das böse Wollen, become public benefits, ${ }^{30}$ werden öffentliche Reichtümer, Tugenden, also Produktivitäten, wie immer Sie benefits übersetzen wollen. »[D]er Teufel ist ein Egoist «, sagt dann Faust zu Mephisto, »Und tut nicht leicht um Gottes willen,/Was einem andern nützlich ist «. ${ }^{31}$ Die große Einsicht ist, dass das Böse (Eigennutzenmaximierung, Ausbeuten, Ungerechtigkeit, Egoismus und dergleichen mehr) zuzulassen, also all das, was in der aristotelischen oder christlichen oder islamischen oder jüdischen Tradition, also in der religiösen, ethischen Traditionslinie diskriminiert ist und klein gehalten wird, hinzunehmen und zu lizensieren, gute Effekte freisetzt. Diese mephistophelische Volte wird vom kapitalistischen Wirtschaftssystem und nur vom Wirtschaftssystem lizensiert - und das ist auch gut so.

Ich komme zum Schluss, ich bin emeritiert, genieße das, habe aber immer noch Kontakt zu einigen wenigen BWL- und VWL-Lehrern meiner Uni - man sagt

28 Vgl. Marx, Karl/Engels, Friedrich: »Manifest der Kommunistischen Partei«, in: Marx Engels Werke. Band 4, Berlin: Dietz 1977, S. 459-493, hier S. $463 f$.

29 J.W. Goethe: Faust. Der Tragödie Erster Teil, V. $1335 \mathrm{f}$.

30 Ursprünglich stammt diese Formulierung aus dem Untertitel von Bernard Mandevilles Bienenfabel (vgl. Mandeville, Bernard: The Fable of the Bees: Or, Private Vices, Publick Benefits. The First Volume, Oxford: Clarendon 1924), mit der sich Adam Smith in verschiedenen Schriften auseinandergesetzt hat. Vgl. Smith, Adam: The Theory of Moral Sentiments (= The Glasgow Edition of the Works and Correspondance of Adam Smith, Band 1), herausgegeben von David D. Raphael/Alexander L. Macfie, Oxford: Clarendon 1976; Smith, Adam: An Inquiry into the Nature and Causes of the Wealth of Nations (= The Clasgow Edition of the Works and Correspondance of Adam Smith, Band 2), 2 Bände, herausgegeben von Roy H. Campbell/Andrew S. Skinner/William B. Todd, Oxford: Clarendon 1976. 
immer interdisziplinär und wann ist man es schon mal? -, die mich schwer traumatisiert und angeregt haben. Wenn ich die frage: "Was ist denn sozusagen Eure Philosophie, Euer Theorierahmen, wenn Ihr das so ein bisschen explizit machen wollt?«, dann sagen sie alle: »Kritischer Rationalismus.« Der Name Albert fiel ja schon, Albert als deifizierte Figur der Mannheimer Theorieszene, kritischer Rationalismus. ${ }^{32}$ Und wenn ich dann sage: "Ich finde das aber unplausibel, weil Kapitalismus unkritisch ist", also unkritisch gegenüber dem Bösen, Teil von jener Kraft, die stets das Böse will, weil sie weiß, dass man damit das Gute schafft, reagieren die irritiert. Wenn ich sage: Kapitalismus, geldfixierter Kapitalismus ist unkritischer Irrationalismus und genau deshalb so produktiv und so überzeugend, kriege ich wenig Zustimmung von BWL- und VWL-Professoren, auch wenig Zustimmung von Theologen und Soziologen und, ich bin mir sicher, auch wenig Zustimmung von Ihnen. Aber ich denke, dass ich einigermaßen zäh - es sei denn, Sie haben wirklich sehr gute Argumente - bei dieser These bleiben werde. Ich schließe also mit der Feststellung, dass der Kapitalismus in seinem Verhältnis zum Steuermedium Geld nur (deshalb) als rational dargestellt werden kann, weil er rational und aufgeklärt genug ist, den unkritischen Irrationalismus, der im Finanzwesen und im Geldverkehr steckt, zuzulassen. Das ist die Rationalität des Kapitalismus: Irrationalität zu lizensieren. Vielen Dank, dass Sie meine Zumutungen ertragen haben, ich bin gespannt auf Ihre Fragen.

\section{Literatur}

Albert, Hans: Kritischer Rationalismus, Tübingen: Mohr Siebeck 2000.

Benn, Gottfried: Sämtliche Werke. Band I. Gedichte 1, herausgegeben von Gerhard Schuster, Stuttgart: Klett-Cotta 2007, S. 174.

Bertram, Brigitte/Jocham, Andrea: »Ehe auf Rädern«, in: Handelsblatt vom 07.05.2003, https://www.handelsblatt.com/archiv/fuenf-jahre-daimler-chrysl er-ehe-auf-raedern/2244874.html?ticket=ST-2757397-thoo6FuY9bn5SSQkDSD $\mathrm{N}$-ap3 (letzter Abruf 20.06.2020).

Binswanger, Hans Christoph: Geld und Magie. Eine ökonomische Deutung von

Goethes Faust, 2. vollständig überarbeitete Ausgabe, Hamburg: Murmann 2005.

Deutschmann, Christoph: Die Verheißung des absoluten Reichtums. Zur religiösen

Natur des Kapitalismus, 2., überarbeitete Auflage, Frankfurt a.M./New York: Campus 2001. 
Goethe, Johann Wolfgang von: Faust. Der Tragödie Erster Teil, in: Ders., Faust. Texte (= Frankfurter Ausgabe, Band 7,1), herausgegeben von Albrecht Schöne, Frankfurt a.M.: Deutscher Klassiker Verlag 1994, S. 33-199.

Goethe, Johann Wolfgang von: Faust. Der Tragödie Zweiter Teil, in: Ders., Faust. Texte (= Frankfurter Ausgabe, Band 7,1), herausgegeben von Albrecht Schöne, Frankfurt a.M.: Deutscher Klassiker Verlag 1994, S. 201-464.

Hegel, Georg Wilhelm Friedrich: „Differenz des Fichte'schen und Schelling'schen Systems der Philosophie«, in: Ders., Gesammelte Werke. Band 4 (= Jenaer Kritische Schriften), herausgegeben von Hartmut Buchner/Otto Pöggeler, Hamburg: Meiner 1968, S. 1-92.

Hörisch, Jochen: Kopf oder Zahl. Die Poesie des Geldes, Frankfurt a.M.: Suhrkamp 1996.

Hörisch, Jochen: Man muss dran glauben. Die Theologie der Märkte, München: Fink 2013.

Lethen, Helmut: Verhaltenslehren der Kälte. Lebensversuche zwischen den Kriegen, Frankfurt a.M.: Suhrkamp 1994.

Luhmann, Niklas: Soziale Systeme. Grundriss einer allgemeinen Theorie, Frankfurt a.M.: Suhrkamp 1984.

Luhmann, Niklas: Die Wirtschaft der Gesellschaft. Frankfurt a.M.: Suhrkamp 1988. Luhmann, Niklas: Die Kunst der Gesellschaft, Frankfurt a.M.: Suhrkamp 1997.

Luhman, Niklas: Die Religion der Gesellschaft, herausgegeben von André Kieserling, Frankfurt a.M.: Suhrkamp 2000.

Mandeville, Bernard: The Fable of the Bees: Or, Private Vices, Publick Benefits. The First Volume, Oxford: Clarendon 1924.

Marx, Karl: Das Kapital. Erster Band (= Marx Engels Werke, Band 23), Berlin: Dietz 2013.

Marx, Karl: Das Kapital. Dritter Band (= Marx Engels Werke, Band 25), Berlin: Dietz 1983.

Marx, Karl/Engels, Friedrich: »Manifest der Kommunistischen Partei«, in: Marx Engels Werke. Band 4, Berlin: Dietz 1977, S. 459-493, hier S. $463 f$.

Polanyi, Karl: The Great Transformation. Politische und ökonomische Ursprünge von Gesellschaften und Wirtschaftssystemen, übersetzt von Heinrich Jelinek, Frankfurt a.M.: Suhrkamp 1973.

Rühmkorf, Peter: Irdisches Vergnügen in g, Reinbek: Rowohlt 1959.

Simmel, Georg: Philosophie des Geldes (= Gesamtausgabe Band 6), Frankfurt a.M.: Suhrkamp 2011.

Smith, Adam: The Theory of Moral Sentiments (= The Glasgow Edition of the Works and Correspondence of Adam Smith, Band 1), herausgegeben von David D. Raphael/Alexander L. Macfie, Oxford: Clarendon 1976.

Smith, Adam: An Inquiry into the Nature and Causes of the Wealth of Nations (=The Glasgow Edition of the Works and Correspondence of Adam Smith, Band 2), 
2 Bände, herausgegeben von Roy H. Campbell/Andrew S. Skinner/William B. Todd, Oxford: Clarendon 1976.

Vogl, Joseph: Das Gespenst des Kapitals, Zürich: Diaphanes 2010.

Weber, Max: »Die protestantische Ethik und der Geist des Kapitalismus«, in: Ders., Gesammelte Aufsätze zur Religionssoziologie I, Tübingen: Mohr Siebeck 1988, S. 17-206.

Zöllner, Frank: »Der teuerste Flop der Welt?«, in: Die Zeit vom 03.01.2019, https: //www.zeit.de/2019/02/salvator-mundi-leonardo-da-vinci-gemaelde-verkauf (letzter Abruf 20.06.2020). 
